\title{
INFLUENCIA DEL AMBIENTE EN LA MOTIVACIÓN Y LA ATENCIÓN DE LOS ESTUDIANTES PARA EL APRENDIZAJE DE LA QUÍMICA
}

\section{INFLUENCE OF THE ENVIRONMENT IN THE MOTIVATION AND ATTENTION OF STUDENTS FOR THE LEARNING OF CHEMISTRY}

\author{
Fredy Colpas Castillo ${ }^{1}$, Arnulfo Tarón Dunoyer ${ }^{2}$, Rafael González Cuello ${ }^{3}$
}

\begin{abstract}
${ }^{1}$ Ingeniero Químico, M.Sc., Profesor investigador, Facultad de Ciencias Exactas y Naturales, Departamento de Química, Grupo de investigación Carboquímica. Universidad de Cartagena, Campus de San Pablo, Cartagena-Bolívar, Colombia, e-mail:

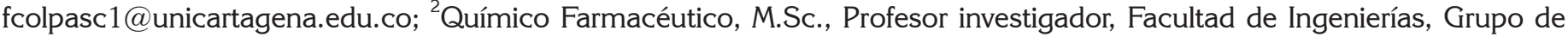
GIBAE. Universidad de Cartagena, Av. del Consulado Calle 30 \# 48-152, Cartagena-Bolívar, e-mail: atarond@unicartagena. edu.co; ${ }^{3}$ Microbiólogo de Alimentos, Ph.D., Facultad de Ingenierías, Grupo de GIBAE. Universidad de Cartagena, Av. del Consulado Calle 30 \# 48-152, Cartagena-Bolívar, e-mail: rgonzalezc1@unicartagena.edu.co
\end{abstract}

Rev. U.D.C.A Act. \& Div. Cient. 21(1): 227-233, Enero- Junio, 2018

https://doi.org/10.31910/rudca.v21.n1.2018.681

\section{RESUMEN}

Diversos investigadores sostienen que solo a través de una conciencia de las diferencias y de las similitudes entre la educación, el ambiente y las neurociencias será posible lograr una fundamentación común necesaria para una ciencia integrada de la educación, el cerebro, la mente y el aprendizaje. El objetivo de la investigación fue determinar si el ambiente de aprendizaje y la neurociencia influyen en la atención y en la motivación de los estudiantes, que traen, como consecuencia, cambios en el rendimiento académico. El experimento, se realizó con dos grupos de estudiantes universitarios, separados en aulas, con diferente ambiente de aprendizaje, que cursaban la asiganatura química general, donde el $76 \%$ fueron de género masculino y el $24 \%$, femenino, para el grupo 1 y el $60 \%$, masculino y $40 \%$ del sexo femenino, para el grupo 2, con edades comprendidas entre loas 17 y 21 años, para ambos grupos. Para evaluar la capacidad de atención, se empleó el test d2 elaborado por Brickenkamp Zillmer y para el análisis de la influencia de los ambientes de aprendizaje, se diseñó un test tipo Likert. El primer grupo, se le realizó el ejercicio docente en un ambiente convencional, el segundo grupo trabajó en ambientes no convencionales, con docentes formados para la aplicación de los conceptos de neurociencia. Los resultados mostraron que existe una relación estadísticamente significativa entre los resultados, respecto al rendimiento académico, para los dos grupos, a un nivel de confianza del 95\%, lo cual, es importante para tener en cuenta en futuros proyectos de educación, que permitan una verdadera transformación en la enseñanza de la química, en programas de ingenierías.
Palabras clave: Ambiente de aprendizaje, rendimiento académico, química, motivación.

\section{SUMMARY}

Many researchers stated that only through conscience about differences and similarities on education, environment and neurosciences it will be possible to achieve a solid base for get an integrated science including the education, brain, mind and learning. The objective of this study was to determine if the learning environment and neuroscience influence the attention and motivation of engineering students, resulting in changes on academic performance. The tests were conducted employing two university students groups taking the subject general chemistry of which $75 \%$ were male and 25\% female, with ages between 17 and 21 years. It is important to clear that both students groups had different learning environment. The first group was taught in conventional learning environments, the second one was taught in a non-conventional environments, with teachers trained to apply the concepts of neuroscience. The Brickenkamp - Zillmer test $\mathrm{d} 2$ was employed to evaluate the student's attention capacity while the Likert test was used in order to evaluate the influence of learning environments. The results showed that there is a statistically significant relationship between the results with respect to academic performance, for both groups which is important to take into account in future education projects that allow a real transformation teaching of chemistry in engineering programs. 
Key words: Learning environment, academic performance, chemistry, motivation.

\section{INTRODUCCIÓN}

El rendimiento académico de los estudiantes de la asignatura química general de la Universidad de Cartagena, históricamente, han presentado un alto pocentaje de alumnos no aprobados, de acuerdo con los datos reportados por la oficina de registro académico; esta situación impulsa evaluar las causas que influyen en los bajos rendimientos de esta asignatura. Los factores internos, como la motivación y la atención, tienen una papel relevante en el proceso de enseñanza-aprendizaje.

Investigaciones realizadas muestran una despoblación de estudiantes en las carreras de química de diversas universidades, de todo el mundo (Navarro \& Casero, 2012; González et al. 2002); es así, como Blakemore \& Frith (2005) afirman que, tal vez, el objetivo de la educación para los adolescentes debería cambiar e incluir un refuerzo de control interno, esto es, por ejemplo: un aprendizaje autorregulado, cierta evaluación crítica del conocimiento transmitido y habilidades de metaestudio. Es justamente esta serie de comportamientos los que han de enseñárseles a los alumnos que ingresan a la Universidad (Bourgeois et al. 2016).

De igual forma, muchos de los problemas a los que se enfrentan las universidades, se deben a las escasas prácticas docentes que fomenten el desarrollo y la generación de conocimiento en el estudiante; igualmente, la falta de ambientes de aprendizaje con motivación, a consecuencia del desconocimiento del funcionamiento del cerebro, entre otros aspectos, que pueden influir en la adquisición y en el desarrollo de habilidades de orden superior (Martínez, 2013).

Gargallo et al. (2014) encontró que, en los últimos años, los alumnos de las preparatorias de la Universidad Autónoma de Nuevo León presentan deficiencias en la comprensión de los conceptos matemáticos, implícitos en los enunciados de problemas de física, en todos los niveles de enseñanza de esta materia, por lo que manifiesta que la diferencia entre lo que se enseña y lo que se aprende es mayor de lo que los profesores tienen conciencia (Gargallo et al. 2014).

Uno de los aspectos fundamentales es la preocupación por la búsqueda de motivación del alumno, pues una una gran parte de los fracasos en las ciencias tienen su origen en un posicionamiento inicial afectivo totalmente destructivo de sus propias potencialidades en este campo, que es provocado, en muchos casos, por la inadecuada introducción por parte de los maestros" (Lewthwaite \& Wulf, 2017).
Rivera Michelena (2016) planteó que un individuo está en capacidad de aprender cuando al colocarlo en la zona de desarrollo próximo, es capaz de construir conocimiento, teniendo en cuenta la influencia del medio social. Belloch (2012) señala que la comunicación en el proceso de enseñanza y aprendizaje se ha transformado con el uso de las TIC, dándose de dos formas, sincrónica y asincrónicamente. La primera situación implica que la educación se realiza en el mismo tiempo y espacio, correspondiente al aula tradicional; hay una variante a esta práctica, que consiste en realizar el acto educativo en el mismo espacio, pero en tiempos diferentes, caso del aula tradicional a distancia (Escalona Tapia \& Fontal Rivera, 2016; Pérez et al. 2012).

Por otro lado, parece existir inquietud y preocupación entre los docentes y las autoridades universitarias, ya que se han comenzado a plantear si estas carencias de motivación de los estudiantes por continuar sus estudios y quizá también su desinterés por iniciarlos, no guarda una estrecha relación con algunas limitaciones y deficiencias de la enseñanza que se les ofrece y con los bajos resultados académicos que obtienen, particularmente, en los cursos introductorios (Vázquez-Alonso \& Manassero-Más, 2016).

Según la teoría del localizacionismo cerebral, la actividad matemática y de las ciencias física y la química, se presenta, en mayor medida, en el lóbulo frontal y parietal del cerebro (Fernández Bravo, 2010). El mismo autor menciona, que dentro del lóbulo parietal, se registra mayor consumo de energía con la actividad matemática, en la región denominada surco intraparietal y en la región inferior. Parece ser que la región inferior parietal controla el pensamiento matemático y la capacidad cognitiva visual-espacial.

Actualmente, se cree que las tareas complejas del procesamiento matemático se deben a la interacción simultánea de varios lóbulos del cerebro. En el cerebro encontramos la respuesta para la transformación y es en él donde ocurrirá la transformación: en el cerebro del maestro y en el cerebro del alumno (Campos, 2010)Lejos de que las Neurociencias se caractericen como una nueva corriente que entra al campo educativo o que se transformen en la salvación para resolver los problemas de aprendizaje o de la calidad de la educación, la propuesta es que sea una ciencia que aporte nuevos conocimientos al educador, así como lo hace la Psicología, por ejemplo, con el propósito de proveerle de suficiente fundamento para innovar y para transformar su práctica pedagógica (Campos, 2014).

El objetivo de la cognitiva es comprender cómo emergen las actividades cognitivas de operaciones biológicas en el tejido neural (Vahey et al. 2017; De Houwer et al. 2013; Van Duijvenvoorde et al. 2016). Vale la pena recordar que, en este proceso de vincular los aportes neurocientíficos al aprendiza- 
je, se necesita diferenciar lo que ya está validado, lo que aún son hipótesis o probabilidades, lo que es mera especulación o mito y, por fin, diferenciar las generalizaciones equivocadas que se hacen, debido a una comprensión limitada del tema (Campos, 2014).

Desde hace siglos, se sabe que en el cerebro se produce la acción intelectual; un complejo proceso que guarda todavía, para nuestro conocimiento, enigmas y secretos de insospechada magnitud, en cantidad y en tamaño. En 1906, los investigadores Camilo Golgy y Santiago Ramón y Cajal descubrieron la ramificación de las células nerviosas, sus conexiones o sinapsis. A partir de ese momento, el avance neurocientífico ha sido relevante, aunque actualmente estemos lejos de dar respuestas cómo funciona el cerebro; es tarea educativa principal incorporar a la actividad pedagógica lo que sabemos sobre el "cómo pensamos" y "cómo sentimos" y traer, a consideración, algunas preguntas (Fernández Bravo, 2010) Es mportante mencionar que, a través de un enfo- que metodológico pertinente, se puede indagar los significados de las acciones y de los pensamientos de los docentes, porque "se refiere en el más amplio sentido a la investigación que produce datos descriptivos sobre las propias palabras habladas o escritas y el comportamiento observable de las personas" (Figueroa et al. 2016; Taylor et al. 2016).

\section{MATERIALES Y MÉTODOS}

Población de estudio: La población objeto de estudio para esta investigación fueron dos grupos de 25 de estudiantes de la educación superior, universitarios separados en aulas con diferente ambiente de aprendizaje, que cursaban la asiganatura química general, de los cuales, el $76 \%$ fueron de género masculino y el $24 \%$, femenino, para el grupo 1 y el $60 \%$, masculino y $40 \%$, del sexo femenino, para el grupo 2 , con edades comprendidas entre los 17 y 21 años, para ambos grupos. En la tabla 1, se describe las condiciones y las caracteriasticas de la población, objeto de estudio.

Tabla 1. Condiciones y caracteriasticas de la población objeto de estudio.

\begin{tabular}{|c|c|c|c|c|}
\hline \multicolumn{5}{|c|}{ Caracteristicas y condicones de la población estudiantil } \\
\hline Grupo de estudio & $\begin{array}{c}\text { Ambiente de aprendi- } \\
\text { zaje }\end{array}$ & $\begin{array}{c}\text { Estudiantes } \\
\text { hombres }\end{array}$ & $\begin{array}{c}\text { Estudiantes mu- } \\
\text { jeres }\end{array}$ & $\begin{array}{c}\text { Edad promedio } \\
\text { (años) }\end{array}$ \\
\hline Grupo 1 & Convencional & 19 & 6 & 19 \\
\hline Grupo 2 & no convencional & 15 & 10 & 19 \\
\hline
\end{tabular}

Evaluación de la capacidad de atención: Para evaluar la capacidad de atención sostenida y selectiva en los grupos de estudiantes, se utilizó el test d2, elaborado por Brickenkamp y Zillmer, en 1981 (Brickenkamp \& Zillmer, 2002), con el fin de garantizar que la muestra seleccionada, tendría iguales condiciones iniciales, referente a la capacidad de atención de los estudiantes.

Caracteristicas de los ambientes: En el grupo 1, se utiliza un ambiente convencional (tradicional), en el que se aplican herramientas o instrumentos de evaluación, como son el test d2, cuestionario tipo Likert y, como variable de respuesta, las calificaciones reportadas en los registros académicos de los estudiantes y en el grupo 2, los estudiantes son acompañados con docentes informados para realizar su proceso de enseñanza, con prácticas pedagógicas que influyen en los factores internos de los estudiantes, como son la atención y la emoción, en formas diferentes, en donde se tiene en cuenta los conceptos de neurociencia y el regisro de calificaciones, como variable de respuesta.
Aprendizaje: Para el análisis de la influencia de los ambientes de aprendizaje, la motivación y la práctica docente, se diseñó un test tipo Likert. El cuestionario fue aplicado a estudiantes, con nivel académico similar, sin antecedentes en su hoja de vida, referentes a problemas que pudiesen afectar su desempeño estudiantil (Tabla 2).

Los instrumentos utiizados durante la investigación para la recoleccion de los datos, se resumen en la tabla 3.

\section{RESULTADOS Y DISCUSIÓN}

Los resultados de las calificaciones obtenidas por los estudiantes y reportadas en los registros académicos de los dos grupos, muestran diferencias en sus valores. Estas diferencias podrían ser consecuencia de los cambios realizados en el ambiente de aprendizaje y la pedagogía del docente, durante las pruebas de la investigación (Figura 1). Para comprobar estas influencias, se realizó prueba estadísica t-student; para la homogenización de las muestras, inicialmente, 
Tabla 2. Instrumentode medición tipo LIKERT para evaluar actitudes para el aprendizaje en diferentes ambientes de aprendizaje.

Responder con una X cada una de las siguientes preguntas teniendo en cuenta la siguiente escala de calificación: 5- Muchísimo. 4- Mucho. 3- Me es indiferente. 2- poco. 1- muy poco.

\begin{tabular}{|c|c|c|c|c|c|c|}
\hline & Pregunta & 5 & 4 & 3 & 2 & 1 \\
\hline 1 & ¿Qué tanto le agradó la clase de química general? & & & & & \\
\hline 2 & ¿Se sentía incómodo durante la clase de química general? & & & & & \\
\hline 3 & ¿Le dieron ganas de salir del salón para no escuchar la clase de química general? & & & & & \\
\hline 4 & ¿El ambiente del salón de clase ayuda al desarrollo del proceso de aprendizaje? & & & & & \\
\hline 5 & $\begin{array}{l}\text { ¿En el inicio de la clase el profesor realiza charla que ayuda a interesarte sobre el tema a } \\
\text { desarrollar? }\end{array}$ & & & & & \\
\hline 6 & ¿Sentía sudoración durante el desarrollo de la clase? & & & & & \\
\hline 7 & $\begin{array}{l}\text { ¿Utiliza el profesor ayudas educativas y redes sociales para el proceso de enseñanza- } \\
\text { aprendizaje? }\end{array}$ & & & & & \\
\hline 8 & ¿El profesor de química general es interactivo con los estudiantes? & & & & & \\
\hline 9 & ¿Le parece que la clase de química general es aburrida? & & & & & \\
\hline 10 & ¿Sentía alegría deseos de participar durante la clase? & & & & & \\
\hline
\end{tabular}

Tabla 3. Instrumentos utilizados para la recolección de la información.

\begin{tabular}{|c|c|}
\hline \multicolumn{2}{|c|}{ Instrumentos de evaluación } \\
\hline Atención y velocidad de procesamiento & Test de homogeneidad d2 \\
\hline Motivación & Cuestionario tipo Likert \\
\hline Desempeño académico & Registro de calificaciones \\
\hline
\end{tabular}

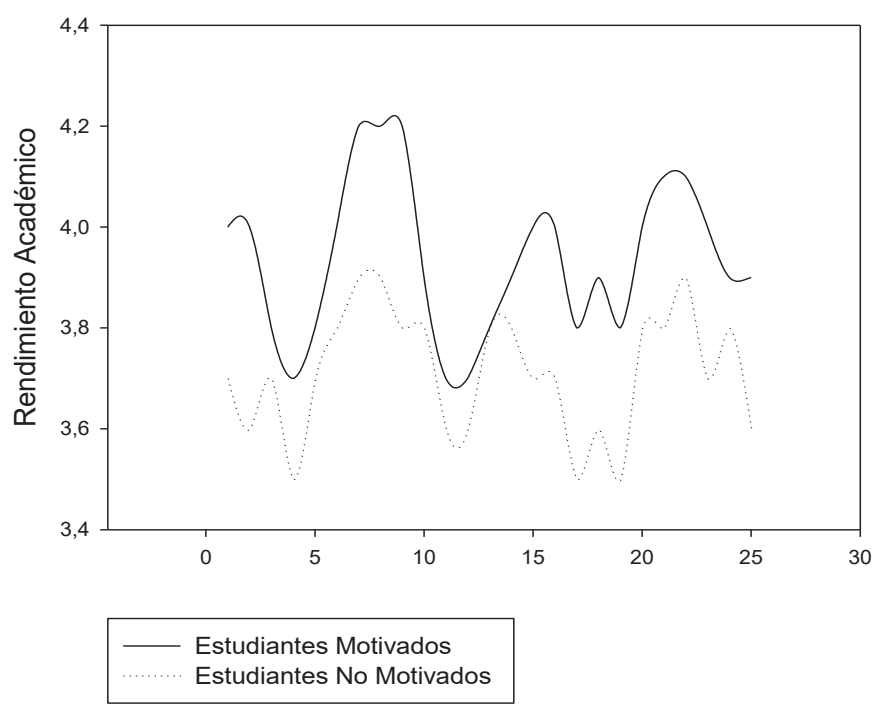

Figura 1. Comparación del rendimiento académico de los dos grupos de estudiantes. 
se realizó el test d2, que mide la capacidad de atención sostenida y selectiva. La atención selectiva y la concentración mental, se reflejan en tres componentes del d2, como son, la velocidad o cantidad de trabajo, calidad de trabajo y la relación entre velocidad y la precisión de actuación.

Para el analisis de la motivación, se diseño test tipo Likert, buscando respuestas relacionadas en cuatro grandes aspectos: la relación ambiente-motivación, la relación ambiente-atención, práctica docente-motivación y práctica docente-atención. Se aplicó los instrumentos de valoración a los dos grupos de estudio, teniendo como variable de respuesta, el rendimiento académico de los estudiantes. La evaluación de la motivación, se realizó a través de preguntas de tipo liker. Los resultados de los instrumentos de medición y los registros de calificaciones fueron tabulados, para la realización del análisis estadístico. Teniendo en cuenta el número de datos y el tipo de estudio, se aplicó una prueba t-student, para muestras independientes, suponiendo igualdad de varianzas, por tratarse de un análisis transversal, con variable aleatoria numérica y un valor de alfa de 0.005 (Tabla 4).

Tabla 4. Resultados de la prueba t-student para el analisis de diferencias significativas entre los resultados de las evaluaciones académicas.

\begin{tabular}{|c|c|c|}
\hline Medidas Estadisticas & Variable 1 & Variable 2 \\
\hline Media & 3,9360 & 3,712 \\
\hline Varianza & 0,0232 & 0,0152 \\
\hline Observaciones & 25,0000 & 25 \\
\hline Grados de libertad & 48,0000 & \\
\hline $\mathrm{P}(\mathrm{T}<\mathrm{t})$ una cola & 0,00000035 & \\
\hline $\mathrm{P}(\mathrm{T}<\mathrm{t})$ dos colas & 0,00000070 & \\
\hline
\end{tabular}

El analisis estadístico, se realizó mediante una prueba t-student, para muestras independientes y varianzas iguales, teniendo en cuenta la normalización de los datos y la naturaleza de las muestras.

Los resultados encontrados en esta investigación son coherentes con los reportados por De la Barrera \& Donolo (2009), quienes manifiestan que los neurotransmisores dopamina, como acetilcolina, incrementan los aprendizajes en los estudiantes. Igualmente, la investigación soporta la premisa de Beltrán Llera (2000), que es donde influye el estilo y la inteligencia, ingredientes básicos de la creatividad; establece que todos los alumnos tienen, al menos, cierto potencial para ser creativos y las diferencias radican en el grado en que utilizan dicho potencial.

La prueba demuestra que sí existe diferencia significativa entre los resultados, para un valor $\alpha=0.005$, lo que indica que el grupo de estudiantes, objeto de estudio, presentaron un cambio en la motivación de manera positiva, cuando se llevaron a un salón de clase con ambientes de aprendizaje motivacionales y diferentes a los tradicionales, con docentes formados en el area de la neurociencia y se refleja en un mayor rendimiento académico.
Podemos considerar que el desconocimiento y la no aplicación por parte del docente, de conceptos de neurociencia, incide en la falta de atención y la motivación, ya que estos factores internos están directamente relacionados con el funcionamiento del cerebro y facilitan el proceso de aprendizaje.

La motivación juega un papel transcendental en el proceso de enseñanza aprendizaje, tanto para el alumno como para el docente. Es relevante resaltar la importancia que tiene en un estudiante al adquirir competencias en un campo determinado, que lo haga con agrado o que el ambiente de aprendizaje sea el más adecuado.

Para la enseñanza, a nivel unversitario, es necesario relacionar las ciencias del aprendizaje y la investigación cerebral y asi comprender y entender el aprendizaje en los seres humano y poder avanzar en los procesos de enseñanza y aprendizaje, de todas las ciencias. Análogamente, buscar ambientes de aprendizaje que tengan en cuenta las características funcionales del cerebro, para implementar estrategias didácticas, que sean compatibles con la naturaleza biológica del aprendizaje.

Es importante considerar, en posteriores investigaciones, factores, tales como: el nivel socioeconómico de los parti- 
cipantes, situación laboral o condiciones personales, situaciones familiares, diferentes perfiles de los sujetos de estudio y aumentar el tiempo de exposición durante las prácticas docentes.

Agradecimientos: A todos los miembros de los grupos de investigación GIBAE de la Universidad de Cartagena. Conflicto de intereses: El manuscrito fue preparado y revisado con la participación de todos los autores, quienes declaramos que no existe conflicto de intereses que ponga en riesgo la validez de los resultados presentados.

\section{BIBLIOGRAFÍA}

1. BELLOCH, C. 2012. Las tecnologías de la información y comunicación (TIC) en el aprendizaje. Valencia. (España). Universidad de Valencia. 11p.

2. BELTRÁN LLERA, J. 2000. Intervensión psicopédagogica y curriculum escolar. Ed. Piramide. (Madrid). 400p.

3. BLAKEMORE, S.; FRITH, U. 2005. Target article with commentaries: the learning brain: lessons for education: a précis. Developmental Science.(Reino Unido). 8(6):459-471.

4. BOURGEOIS, A.; CHELAZZI, L.; VUILLEUMIER. 2016. How motivation and reward learning modulate selective attention. Progress in Brain Research. (Netherlands). 229:325-342.

5. BRICKENKAMP, R.; ZILLMER, E. 2002. Test de Atención d2. Madrid: TEA Ediciones.

6. CAMPOS, A. 2010. Neuroeducación: uniendo las neurociencias y la educación en la búsqueda del desarrollo humano. La Educ@ación. (Colombia).143:1-14.

7. CAMPOS, A. 2014. Los aportes de la neurociencia a la atención y educación de la primera infancia. (Bolivia). Disponible desde Internet en: http://www.unicef. org/bolivia/056_NeurocienciaFINAL_LR.pdf (con acceso el 04/11/2017).

8. DE HOUWER, J.; BARNES-HOLMES, D.; MOORS, A. 2013. What is learning? On the nature and merits of a functional definition of learning. Psychen Bull Chen. 20(4):631-642.

9. DE LA BARRERA, M.; DONOLO, D. 2009. Neurociencias y su importancia en contextos de aprendizaje. Rev. Digital Universitaria. (México). 10(4):1-18.
10. ESCALONA TAPIA, J.; FONTAL RIVERA, B. 2016. Cambio en las teorías científicas desde la visión de estudiantes de educación en la Universidad de los Andes, Venezuela. Rev. U.D.C.A Act. \& Div. Cient. (Colombia). 19(2):467-477.

11. FERNÁNDEZ BRAVO, J.A. 2010. Neurociencias y Enseñanza de la Matemática. Prólogo de algunos retos educativos. Rev. Iberoamericana de Educación. (España). 51(3):1-12.

12. FIGUEROA, R.; SALAZAR, C.; BERNAL, M. 2016. Conocimiento pedagogico en docentes de investigación formativa en la Univeesidad del Atlantico. Revista Tecné, Episteme y Didaxis: TED. Año 2016, Número Extraordinario. Memorias, Séptimo Congreso Internacional sobre Formación de Profesores de Ciencias. 12 al 14 de octubre de 2016, Bogotá.(Colombia). p.1-12.

13. GARGALLO, B.; BERTOMEU, I.; CHORNET, S.; OLMEDO, C.; NAVAL, S. 2014. Metodología centrada en el aprendizaje. Su impacto en las estrategias de aprendizaje y en el rendimiento. Rev.Española de Pedagogía. (España). 259:415-435.

14. GONZÁLEZ, M.C.; ÁLVAREZ, P.; CABRERA, L.; BETHENCOURT, J. 2002. La toma de decisiones académicas del estudiantado de la Universidad de La Laguna en la elección de los créditos de libre configuración. Contextos Educativos. 5:123-140.

15. LEWTHWAITE, R.; WULF, G. 2017. Optimizing motivation and atention for motor performance and learning. Current Opinion in Psychology. (Reino Unido). 16:38-42.

16. MARTíNEZ, B.H. 2013. Causas del bajo rendimiento escolar origina un alto nivel de deserción escolar y habilidades para estudiar ayudan a mejorar el rendimiento escolar. Rev. Iberoamericana para la investigación y el desarrollo educativo. (España). 2:1-17.

17. NAVARRO, C.; CASERO, A. 2012. Análisis de las diferencias de género en la elección de estudios universitarios. Estudios sobre educación. (España). 22:115-132.

18. PÉREZ, L.; ANUAR, C.; SAKER, F. 2012. Importancia del uso de las plataformas virtuales en la formación superior para favorecer el cambio de actitud hacia las TIC. Rev. Iberoamericana de Evaluación Educativa. (Colombia). 6(1):153-166. 
19. RIVERA MICHELENA, N. 2016. Una óptica constructivista en l a búsqueda de soluciones pertinentes a los problemas de la enseñanza-aprendizaje. /learning problems. Educ. Med. Super. (Cuba). 30(3):609-614.

20. TAYLOR, S.J.; BODGAN, R.; DEVAULT, M.L. 2016. Chapter Introduction: Go to the People. En: Introduction to Qualitative Research Methods: A Guide and Resource. (4a Ed.). John Wiey \& Sons: Nueva Jersey. p.1-26.

21. VAHEY, N.; BENNETT, M.; WHELAN, B. 2017. Conceptual advances in the cognitive neuroscience of learning: Implications for relational frame theory. J. Contextual Behavioral Science. (Holanda). 6(3):308-313.
22. VAN DUIJVENVOORDE, A.; PETERS, S.; BRAAMS, B.; CRONE, E. 2016. What motivates adolescents? Neural responses to rewards and their influence on adolescents' risk taking, learning and cognitive control. Neuroscience and Biobehavioral Reviews.(Reino Unido). 70:135-147.

23. VÁZQUEZ-ALONSO, Á.; MANASSERO-MÁS, M.A. 2016. La voz de los estudiantes de primer año en seis países: evaluación de sus experiencias. Ciéncia \& Educaçâo. (Brasil). 22(2):391-411.

Recibido: Octubre 23 de 2017

Aceptado: Mayo 27 de 2018

Cómo citar:

Colpas Castillo, F.; Tarón Dunoyer, A.; González Cuello, R. 2018. Influencia del ambiente en la motivación y la atención de los estudiantes para el aprendizaje de la química. Rev. U.D.C.A Act.\& Div. Cient. 21(1): 227-233. 